\title{
Los Sistemas de Relaciones Laborales. Análisis desde el Cono Sur de América Latina
}

Jorge Notaro(*)

Resumen: El objetivo de este documento es construir un marco teórico metodológico para el análisis de los Sistemas de Relaciones Laborales teniendo en cuenta las particularidades de la región. Incluye el perfil del sistema y sus interrelaciones con el contexto social, económico y político.

Palabras clave: Metodología, Relaciones laborales, Negociación colectiva.

Abstract: The purpose of this document is to build a methodology for the analysis of the Labor Relations Systems considering the specificity of the region. It includes the system profile and its interactions with the social, economic and political context.

Key words: methodology, labor relations, collective bargaining.

(*) Professor e Pesquisador — Facultad de Ciencias Económicas y de Administración - UDeLaR, Uruguay. E-mail: $<$ jnotaro@netgate.com.uy > . Jorge Notaro WEB: <w ww.jorgenotaro.com $>$. Recebido em 24.8.2010 e aceito em 15.4.2011. 


\section{INTRODUCCIÓN}

El objetivo de este documento es construir un marco teórico metodológico para el análisis de los Sistemas de Relaciones Laborales (SRRLL) de los países del Cono Sur de América Latina, lo que comprende identificar sus características y los procesos de cambio. Se definen las categorías básicas y los indicadores que las miden, se presentan distintos enfoques y se explicitan hipótesis sobre las interrelaciones entre las categorías. Algunas conclusiones de la bibliografía revisada que se refieren a otras coordenadas históricas se convierten en hipótesis de investigación para los países del Cono Sur de América Latina a principios del siglo XXI, sobre su eventual vigencia en cada país y para la región. Las referencias a casos cumplen el papel de ejemplos que ilustran las consideraciones más generales sin adjudicarles una función de verificación de las hipótesis. El objeto de estudio se define en el Glosario elaborado por la OIT como (tradución propia):

Las relaciones individuales y colectivas entre trabajadores y empleadores en el trabajo y surgidas del trabajo, así como las relaciones entre los representantes de trabajadores y empleadores en el nivel de una actividad económica o en el nivel nacional, y su interacción con el Estado. Estas relaciones abarcan aspectos legales, económicos, sociológicos y fisiológicos e incluyen los siguientes temas: selección, contrato, localización, capacitación, disciplina, ascenso, despido, finalización del contrato, salarios, horas extras, bonos, participación en las utilidades, educación, salud, seguridad, recreación, vivienda, duración del trabajo, descanso, vacaciones y seguros de desempleo, enfermedad, accidentes, maternidad, vejez e incapacidad. (ARRIGO \& CASALE, 2005, p. 163).

Este artículo se limita a los aspectos macrosociales considerando los actores colectivos y el derecho colectivo, la macroeconomía y el sistema político. Se tienen en cuenta las especificidades de los países del Cono Sur de América Latina desde la segunda mitad del siglo XX, es decir: (I) la inestabilidad política que se manifestó en períodos de dictaduras que se alternaron con períodos de democracia, y en estos últimos, cambios de partidos de gobierno que se tradujeron en las políticas públicas; (II) la inestabilidad económica que se manifestó en períodos de crecimiento que se alternaron con profundas crisis; (III) los cambios en la estructura social por la sustitución de importaciones que desarrolló la clase obrera urbana, la migración rural-urbana que alimentó un sector informal, la participación del Estado que desarrolló la burocracia y las medidas de apertura, liberalización y desregulación de la década de los noventa que resultaron en la contracción de la actividad industrial, el cierre de numerosos grandes establecimientos, la reducción del número de obreros y el aumento del trabajo no registrado.

Se presentan las principales características del enfoque y los componentes del SRRLL. Las interacciones del SRRLL con el contexto social, económico y político se abordan desde dos ángulos complementarios, los del contexto sobre el SRRLL que se consideran condicionantes y los del SRRLL sobre el contexto que se llaman impactos o resultados.

\section{Premisas metodológicas}

Las cinco corrientes que constituyen las principales fuentes de este artículo son los trabajos de Dunlop (1958) y los posteriores en colaboración con Kerr, Harbison y Myers 
(1960, 1971); la conocida como de las "opciones estratégicas de los actores" (Kochan, Mc. Kersie y Cappelli 1983), Kochan y Katz (1988), Kochan y Piore (1990); Kochan, Katz y Mc Kersie (1993), Kochan, Locke y Piore, eds. (1995); los autores que incorporan el análisis de las relaciones de poder entre los actores y de estos con el sistema político, como Hyman (1989) y Touraine (1987, a y b); el pensamiento latinoamericano canalizado en la Asociación Latinoamericana de Sociología del Trabajo que produjo un Tratado latinoamericano de sociología del trabajo (de la Garza [coord.], 2000), presentó su producción en seis congresos y la difundió con la Revista Latinoamericana de Estudios del Trabajo; finalmente, los aportes de la OIT sobre América Latina (OIT 1962, 1978 y 1981); Cardoso y Gindin, (2009) y el MERCOSUR (RELASUR, 1995). Sin perjuicio de la incorporación de aportes de otros autores en diversas oportunidades.

Los SRRLL están asociados al capitalismo ya que suponen que la relación capital-trabajo es la más importante y se justifica como objeto de estudio. Si los trabajadores fueran esclavos o, por el contrario, dueños de los medios de producción que utilizan, las categorías serían otras. Surgieron con la industrialización, convocan a las organizaciones de trabajadores y de empleadores y canalizan el conflicto capital-trabajo. En América Latina a principios del siglo XXI esta definición comprende lo que de la Garza (2010) define como "trabajo clásico", quedando fuera el "trabajo no clásico" definido como "trabajos inmateriales o bien simbólicos objetivados". La construcción de las identidades en el trabajo no clásico, así como las formas de relacionamiento entre los eventuales actores, requieren abordar otro campo de investigación para lograr una perspectiva completa del mundo del trabajo contemporáneo en los países latinoamericanos.

La categoría proceso social orienta este enfoque en tres aspectos. En primer lugar, la búsqueda de las interrelaciones entre los diversos aspectos que estudian las ciencias sociales. Fue uno de los objetivos del informe de OIT (1981: v-vi) sobre las relaciones de trabajo en América Latina: También Hyman (1989) enfatizó sobre la inexistencia de un desarrollo autónomo de los SRRLL y la importancia de las interrelaciones entre los diversos niveles y elementos de la formación social, "capitales nacionales e internacionales y sus diversas fracciones; el Estado y la sociedad civil; relaciones materiales e ideológicas” (HYMAN, 1989, p. 138).

En los países latinoamericanos y los del Cono Sur como parte de estos, es necesario investigar las relaciones con los cambios políticos, económicos y sociales. Cardoso y Gindin (2009) subrayan que los sistemas de relaciones laborales en Argentina, Brasil y México, sin desconocer las diferencias históricas entre los tres países, estuvieron estrechamente ligados al papel del Estado para dar forma a la naturaleza, el ámbito y la dirección del desarrollo social, económico y político.

En los trabajos de la OIT hay una permanente referencia a los cambios en el contexto económico y político, analizando sus interrelaciones con las relaciones laborales. En los últimos años se ha prestado particular atención a los cambios resultantes de los modelos de desarrollo económico, de las dictaduras y el posterior retorno a la democracia, de las crisis económicas y de las políticas de liberalización y apertura (OIT, 1987, p. 7-2 1; RELASUR, 1995, p. 17-24; Cardoso y Gindin, 2009, p. 1-13). Entre los impactos, una preocupación 
central ha sido identificar un sistema de relaciones laborales que estimulara la inversión, el crecimiento y el empleo (OIT 1987: 1).

Un segundo aspecto derivado de la categoría proceso social es la dimensión temporal. El objeto de estudio de las ciencias sociales está en permanente cambio, por lo que una relación entre variables que se cumplía hasta ayer en un país o región podría no cumplirse en otros países y regiones en ese momento así como puede no cumplirse hoy en alguno de los países o regiones. La historicidad de los SRRLL que refleja las particularidades de los países "en desarrollo" ha sido reconocida por las principales corrientes surgidas en los Estados Unidos, que identifica las diferencias en el tiempo y entre países (Kerr, Dunlop, Harbison y Myers, 1971). El enfoque de las "opciones estratégicas" analiza los cambios inducidos por la estructura del PBI y el empleo, las políticas del Presidente Reagan y las estrategias empresariales en sus planes de negocios con repercusiones en las relaciones laborales (Kochan, McKersie y Cappelli, 1983).

En tercer lugar, las especificidades de un lugar durante cierto período se comprenden en un contexto más abstracto y permanente. Se trata de relacionar los planos generales con los concretos y específicos. En un plano general, las relaciones sociales de producción capitalistas implican un antagonismo estructural de intereses entre capital y trabajo que requieren investigar la dinámica de la acumulación de capital, la naturaleza de la clase trabajadora y las formas cambiantes de intervención del Estado en las relaciones entre trabajo y capital (Hyman, 1989). Pero también es necesario tener en cuenta los aspectos particulares (traducción propia):

El estado de las "relaciones industriales" o de la lucha de clase no puede simplemente deducirse de una caracterización general de la coyuntura económica y política, pero tampoco puede entenderse si no se relaciona con este contexto. Es necesario tener en cuenta la especificidad de las instituciones y los procesos de mediación que son en ciertos aspectos peculiares en cada nación, actividad económica, empresa y lugar de trabajo; y cuyos efectos pueden variar en el tiempo (HYMAN, 1989, p. 135).

En cualquier instante de su desarrollo, se considera que un sistema de relaciones industriales está compuesto por ciertos actores, ciertos contextos, una ideología que los mantiene unidos y un cuerpo de normas creadas para dirigir a los actores en el lugar y comunidad de trabajo. (DUNLOP, 1958, p. 19_).

Se excluye el componente de "ideología”, se considera que los actores inciden con sus estrategias en la modificación de los SRRLL aceptando que (traducción propia):

un modelo más realista de las relaciones industriales debe reconocer en primer lugar el papel activo de la administración en dar forma a las relaciones industriales (un punto de vista opuesto al tradicional de reacción frente a las presiones sindicales) y en segundo lugar, los diferentes niveles de la toma de decisiones en las relaciones entre las organizaciones de empresas, trabajadores y gobierno y sus efectos independientes en los resultados de las relaciones industriales. Esta es la razón por la que creemos que el concepto de estrategia, o de opción estratégica, puede ayudar a agregar un componente más dinámico a la teoría de sistemas y ayudar a explicar algunos de los cambios en las relaciones industriales en los Estados Unidos. (KOCHAN, MC KERSIE y CAPPELLI 1983, p. 7-8). 
Es poco frecuente el análisis de las relaciones de poder entre los actores y de estos con el sistema político. Una excepción es el informe de OIT sobre Uruguay, que define los objetivos de los SRRLL y analiza las características del uruguayo (OIT, 1987: 2-5 y 23-41) señalando que:

Una comprensión adecuada de la dinámica de un sistema de relaciones de trabajo supone conocer ante todo la manera como el mismo se fue creando y los factores que determinaron su formación. El sistema refleja los valores y características de un país, tal como fueron descritos en el capítulo anterior, pero también responde a sus propios elementos intrínsecos y en particular a las acciones y actitudes de sus actores. El primero de dichos elementos intrínsecos es, sin duda, la política seguida por el Gobierno con respecto al desenvolvimiento de las relaciones de trabajo. (OIT, 1987, p. 23).

Esta definición incluye la dimensión temporal, las particularidades del sistema y su cambio en relación con el contexto, el papel de los actores incluyendo al Gobierno y sus estrategias.

Como parte del contexto y condicionantes del perfil de los Sistemas y de sus cambios se tiene en cuenta la situación económica, la estructura social y el sistema político. Se incluyen las relaciones de poder en la empresa y con relación al sistema político como condicionantes del SRRLL y como resultado, en aspectos que trascienden al SRRLL como las políticas económicas y la participación de los actores sociales en el sistema político. Con un enfoque similar, Godio y Villarroel (2007) se proponen "Recrear el concepto de Sistema de Relaciones Laborales desde una perspectiva histórica, que contextualice la acción de los Actores Sociales y los productos de su interacción en el actual marco político, económico y social" (GODIO y VILLARROEL, 2007, p. 1).

\section{El Perfil del Sistema}

En los países del Cono Sur de América Latina se pueden diferenciar dos situaciones políticas que imprimen sus características en los SRRLL, la democracia y la dictadura. En lo que sigue se consideran perfiles de Sistemas en contextos democráticos. Se considera que el SRRLL tiene cuatro componentes: los actores, los escenarios, el grado en que se combinan cooperación y participación y confrontación y conflicto y las relaciones de poder. Se consideran "actores" a las organizaciones que representan a los trabajadores o a los empresarios y al Estado. Los "escenarios" comprenden a las instituciones (ámbitos u organismos), a los procedimientos (o formas) de relación de los actores, a las normas legales que definen sus funciones y competencias y a los temas que se discuten (contenidos).

\section{Los actores. Características y estrategias}

El trabajo como actividad humana contribuye a la generación de bienes y servicios y es el punto de partida para la construcción de clases y actores sociales (de la Garza, 2010). Para los trabajadores asalariados así como para los empleadores es necesario diferenciar en el análisis la clase social, definida como los grupos de personas que participan de la 
actividad económica como vendedores o compradores de capacidad de trabajar, de los actores sociales que se generan cuando las personas toman conciencia de su condición, se organizan, explicitan reivindicaciones, tienen capacidad para adoptar medidas de lucha y su representatividad es reconocida por los demás actores.

La constitución de los actores, así como sus opciones estratégicas, están condicionadas por la estructura social, la situación económica y el sistema político. Una primera aproximación es tener en cuenta la importancia relativa de la relación capital-trabajo en el total de ocupados y su evolución con relación a los trabajadores por cuenta propia urbanos y productores familiares rurales. En América Latina el gobierno tiene un papel central como regulador de la negociación colectiva y la huelga, y el poder judicial en la aplicación de las leyes (Cardoso y Gindin, 2009).

Para las organizaciones de trabajadores y de empleadores se tiene en cuenta: a) la cobertura o representatividad según el número de trabajadores o de empresas afiliadas con relación al total de ocupados o de empresas; en los empleadores, también la importancia en el PBI; b) su capacidad de convocatoria; c) los fines y las competencias; d) las corrientes de opinión interna.

En las estrategias es necesario analizar los objetivos económicos y políticos; los más visibles son las aspiraciones de los trabajadores de aumentar el salario real y de los empresarios de aumentar la rentabilidad. Pero también se proponen objetivos en las relaciones de poder, dentro de la empresa y con relación al sistema político.

Se puede hipotetizar que en las organizaciones sindicales las diferencias tienen un fundamento principalmente político y en las organizaciones empresariales responden a fundamentos principalmente económicos.

Los trabajadores asalariados se organizan de distintas formas y en el SRRLL se consideran los sindicatos como expresión de sus intereses y puntos de vista; entre otras organizaciones puede mencionarse las cooperativas, las cajas de auxilio, las policlínicas, los comedores populares, las instituciones culturales y deportivas o los partidos políticos.

Para las organizaciones de empresarios la base social potencial tiene afinidades económicas que resultan de la estructura de la producción y su destino, como agroindustria de exportación o importadores, agro o industria, producción o intermediación financiera, empresas nacionales y transnacionales, de distintos tamaños, dirigidas al mercado interno o al externo, en actividades tradicionales o innovadoras. Esta heterogeneidad dificulta la toma de decisiones del conjunto de las organizaciones empresariales. Las diferencias se manifiestan cuando se relacionan con el sistema político y se expresan en las reivindicaciones sobre el nivel del tipo de cambio, de la tasa de interés, la protección del mercado interno o la promoción de las exportaciones.

El análisis de la estrategia de los actores empresariales en América Latina debe diferenciar dos planos, el de las relaciones laborales y el de la política económica, cuyos impactos pueden tener distinto signo compensando uno los del otro. En el primero se puede hipotetizar que la orientación predominante trata de mantener la subordinación de los trabajadores y reducir los costos de mano de obra. En el segundo los objetivos son 
conservar la propiedad y dar viabilidad a la empresa, aumentar la capacidad de decisión y los campos de acción del empresario. La convergencia de ambos confluye hacia una mayor seguridad y rentabilidad.

En las negociaciones salariales se pueden observar diferencias entre empresas y organizaciones, que resultan de las estrategias globales como, por ejemplo, reducir los costos laborales por hora o aumentar la productividad. Los países de mayor grado de desarrollo son ejemplos de salarios altos por unidad de trabajo que no implican pérdida de competitividad, porque están acompañados por inversiones que incorporan cambios tecnológicos. La reducción del costo unitario (costo de mano de obra por unidad de producto) puede resultar de un mayor nivel de actividad con igual costo de mano de obra o de un nivel de actividad que se reduce en menor ritmo que el costo de mano de obra.

\section{LOS ESCENARIOS}

Los "escenarios" comprenden a quiénes, cómo y qué negocian. Por el papel de la legislación se diferencian los autónomos, los heterónomos delimitados por las normas jurídicas, es decir, "la regulación estatal de las relaciones que se entretejen en torno al factor trabajo" (RELASUR 1995, p. 25) y los mixtos, que incluyen los dos anteriores en forma parcial en distintos aspectos. Según la forma de participación se pueden diferenciar cuatro tipos:

a) Los fundacionales para la negociación colectiva.

b) La participación de las organizaciones de trabajadores y empresarios en organismos oficiales. (OIT, 1981, p. 137-160; OIT, 1987, p. 91-92; RELASUR, 1995, p. 115-120).

c) La participación de los trabajadores en órganos de la empresa. (OIT, 1981, p. 99-117; OIT, 1987, p. 91-92; RELASUR, 1995, p. 121-124).

d) Desde fines del siglo XX, el Diálogo Social y como resultados exitoso del mismo, la concertación y los Pactos (OIT, 1981, p. 83-97; OIT, 1987, p. 73-100). Comprende diversos ámbitos de encuentro y debate, otros actores que se suman a los del tripartismo y nuevos contenidos que abordan aspectos políticos y económicos.

En los dos puntos siguientes se analizarán los fundacionales y el Diálogo Social.

\section{LOS ESCENARIOS FUNDACIONALES}

Se crearon con el objetivo de canalizar el conflicto entre los actores colectivos por la fijación de salarios (Cardoso y Gindin, 2009, p. 17-20 y p. 37-43). La Unión Europea adoptó la Convención de OIT n. 154 (1981) que promueve la negociación colectiva voluntaria y libre adaptada a las características de cada país (Arrigo y Casale, 2005, p. 53).

En la agenda se incluye la negociación colectiva, sus protagonistas, los procedimientos, el nivel y las materias negociadas, la estructura y niveles de articulación, así como los derivados de las cuestiones de encuadramiento de la representación sindical y empresarial. 
En un nivel micro se analizan las técnicas aplicadas a la gestión de la negociación colectiva: visualización del conflicto, organización de reuniones, técnicas de negociación y formas de comunicación interna (OIT, 1981, p. 83-99; OIT, 1987, p. 73-86; RELASUR, 1995, p. 70-94; CARDOSO y GINDIN, 2009, p. 15-36; GODIO y VILLARROEL, 2007).

Por la participación del Estado, se transforman de bi en tripartitos; de acuerdo al grado de centralización los actores pueden ser nacionales, sectoriales, regionales, de empresa o de establecimiento; las actividades urbanas, las rurales y el servicio doméstico tienen tratamientos distintos; por sector institucional se diferencian el privado y el público (OIT, 1987 , p. 147-160).

Se consideran "contenidos" a los problemas o temas que abordan los actores y, si se logran acuerdos, se implementan como convenios o contratos colectivos. Tienen como componente principal los criterios de fijación de salarios y en los últimos tiempos la temática se diversificó en distinto grado según los países y los períodos para dar una respuesta adecuada a los cambios en el contexto.

Los cambios de ritmo de variación de los precios internos y del tipo de cambio, así como los diversos grados de apertura de la economía y de credibilidad de las políticas gubernamentales, se transforman en un problema complejo para las empresas que no quieren perder competitividad por aumento de sus costos de mano de obra y para los trabajadores que no pueden aceptar el deterioro de su salario real.

Es necesario investigar los procedimientos para la fijación de salarios, las condiciones de trabajo, los conflictos y los procedimientos de prevención y resolución. En Argentina, Brasil y México, la fuerte regulación de los sistemas de relaciones laborales transforma a los salarios y otros ingresos en el centro de las negociaciones colectivas (Cardoso y Gindin, 2009, p. 53). Se incorporan como nuevos contenidos la flexibilización y la organización del trabajo durante la década de los años noventa, las condiciones de trabajo de las mujeres y las tercerizaciones o subcontrataciones (CARDOSO y GINDIN, 2009, p. 53-58).

Como hipótesis se pueden considerar las conclusiones de Osaki (2003), según quien las diferencias de los contenidos entre distintos países se explican principalmente por los marcos jurídico-institucionales, las políticas públicas y el contexto económico (traducción propia): "La globalización y el cambio tecnológico afectan la importancia del empleo y la competitividad como temas importantes para los actores sociales, pero la forma en que los abordan está altamente condicionada por los factores señalados anteriormente" (OZAKI, 2003, p. 2).

\section{El Diálogo Social} propia):

El "Diálogo Social" tiene diversas definiciones. En la Unión Europea (traducción

diálogo social es la expresión utilizada para describir los procedimientos de consulta entre los actores socios a nivel europeo. Incluye la discusión, las acciones conjuntas y en algunas oportunidades negociaciones entre los actores europeos socios y 
discusiones entre estos y las instituciones de la Unión Europea. La principal innovación de este concepto es considerar que las organizaciones de trabajadores y de empresarios son socios, a lo que se hace referencia con la expresión "social partners" (ARRIGO y CASALE, 2005, p. 235).

Para la OIT "el diálogo social incluye a la negociación colectiva, a los mecanismos de información y consulta - institucionalizados o no - a los medios participativos y voluntarios de solución de conflictos del trabajo, a la participación — orgánica o inorgánica, en la empresa o en instancias sectoriales y nacionales - y a la concertación social, incluidos los pactos sociales o acuerdos marco, sean bi o tripartitos" (Diálogo social. Concepto y experiencias. Glosario de la Unión Europea. En: <http://europa.eu/scadplus/glossary/ social_dialogue_es.htm $>$ ). La OIT ${ }^{(1)}$ también ha llamado la atención sobre la necesidad de incorporar al Diálogo Social a los actores que no están comprendidos en la relación salarial, ampliando la base social para lograr una mejor representación, promoviendo la superación de las dificultades de organización con programas de fortalecimiento de los actores tradicionales del tripartismo o nuevos. El primer objetivo de las políticas públicas puede ser contribuir a la organización y expresión independientes de los mismos, ayudando a su constitución como actores.

Como condición necesaria es imprescindible considerar que se puede llegar a acuerdos a pesar de los intereses y puntos de vista distintos. Es necesaria la disposición a escuchar de modo que los discursos se entrelacen para que se identifiquen los puntos de acuerdo y de desacuerdo, su importancia relativa y los posibles caminos superadores.

Las interrogantes centrales para los países del Cono Sur se refieren a identificar los escenarios para el Diálogo Social, los actores convocados, los contenidos analizados, los resultados obtenidos y su explicación. En la Unión Europea se espera que el diálogo social contribuya a promover la competitividad y la solidaridad, así como el equilibrio entre flexibilidad y seguridad. ¿Se han logrado estos resultados en los países del Cono Sur de América Latina?

En el MERCOSUR la Declaración Sociolaboral ${ }^{(2)}$ incluye el Diálogo Social entre los derechos y en la primera Memoria elaborada en el 2001, su promoción se priorizó junto con otros cuatro derechos. Como principal cambio de los últimos años se percibe la incorporación de nuevos actores a escenarios de diálogo para el diseño y ejecución de políticas de empleo y sería oportuno evaluar la experiencia.

\section{LA COMBINACIÓN DE CONFRONTACIÓN Y COOPERACIÓN}

La OIT define el conflicto como desacuerdos que generan medidas de lucha y lo diferencia de la disputa que resulta de la incapacidad de llegar a acuerdos (ARRIGO y CASALE, 2005, p. 53) $)^{(3)}$. Se plantea como alternativa para caracterizar la negociación

(1) Conferencia Internacional de Ginebra de 1996

(2) Aprobada por los Presidentes el 10 de diciembre de 1998.

(3) Industrial conflict. Disagreement between labour and management expressed through behaviour such as sabotage, absenteeism and strikes. These specific actions can be distinguished according to their form (organized or unorganized) 
colectiva, el conflicto distributivo o el énfasis en resolver problemas que permitan mejoras para ambas partes.

Se puede considerar que el conflicto se genera cuando cada parte pone énfasis en su enfoque e intereses particulares y se combina en diversos grados con la cooperación/ participación, entendida como la disposición a tener en cuenta el enfoque y los intereses de la otra parte con el fin de lograr acuerdos.

Las experiencias de cooperación/participación y de confrontación/reivindicación, así como la relación de poder entre los actores, se combinan en diversos grados a través del tiempo, en los distintos países y en cada rama de actividad o empresa. Se supone que el conflicto está implícito en las relaciones laborales y que al mismo tiempo pueden existir objetivos comunes de las partes (KOCHAN y KATZ, 1988); el carácter de las relaciones dependerá de la interacción de las estrategias de los actores.

Algunos autores consideran que el mayor grado de cooperación implica una mayor calidad de las relaciones laborales. Para Blanchard y Philippon (2004) la calidad de las relaciones laborales es una definición más general de la calidad del diálogo de los sindicatos tienen con las empresas. Le atribuyen gran importancia para la "velocidad de aprendizaje" de los sindicatos lo que a su vez contribuye, en los países en que los salarios se fijan por negociaciones colectivas, a reducir el desempleo. Construyen dos medidas de la calidad de las relaciones laborales, la primera basada en las huelgas en los años sesenta y la otra en la percepción de los administradores de empresas en los noventa.

Concluyen que "Mostramos que los países con peores relaciones laborales han experimentado mayor y más prolongado desempleo. Mostramos que el efecto permanece aun después de controlado por las instituciones del mercado de trabajo" (BLANCHARD y PHILIPPON, 2004, p. 2). Este enfoque desconoce el conflicto entre capital y trabajo, así como no considera necesario analizar la rapidez de aprendizaje de la administración de las empresas. Probablemente la crisis comenzada en 2008 requiera una revisión de la metodología y las conclusiones.

En América Latina la agenda de investigación incluyó los tipos y causas de los conflictos, el marco jurídico; los procesos de mediación institucional incluyendo la conciliación, el arbitraje y los tribunales de trabajo. Como medidas de lucha se tienen en cuenta la huelga y el cierre patronal (OIT, 1981, p. 161-263; OIT, 1987, p. 121-143; RELASUR, 1995, p. 95-114). Se describe la interacción entre los actores en los procesos de negociación que incluyen conflictos y medidas de lucha (confrontación) y acuerdos (cooperación) en distinto grado y de diversas formas. Se intenta explicarlos en función de las condicionantes contextuales y sus opciones estratégicas. El punto de partida son las demandas y las ofertas de las partes, sucedidos por contraofertas, las medidas de lucha, los procedimientos de resolución de conflictos (conciliación, mediación, arbitraje, negociación), los resultados (costos económicos, sindicalización, grado en que se logran los objetivos) y la evaluación de los actores a posteriori.

and to the party involved. Industrial dispute. Disagreement between labour and management arising from the inability of both parties to resolve their differences. An industrial dispute may result in conflicts such as a strike. The subject matter of a particular dispute may determine whether the dispute is within the scope of industrial legislation and the jurisdiction of a third party (e.g. an industrial tribunal) empowered to assist the parties in resolving the issue(s) concerned by conciliation, mediation and/or arbitration. See Arbitration; Conciliation; Mediation 
La conflictividad se puede considerar creciente según el grado de utilización de medidas de fuerza por alguna o ambas partes, como los paros y huelgas por parte las organizaciones sindicales, o el lockout y los despidos por parte de las organizaciones de empleadores. En algunas ocasiones se pone en marcha una espiral de acciones como los piquetes y las ocupaciones, reprimidos por la intervención policial, a veces con heridos y muertos, lo que origina nuevas movilizaciones contra la represión y nuevos enfrentamientos. El origen de la trasgresión es siempre difícil de identificar y se vuelve casi irrelevante, frente a los costos en vidas humanas y los nuevos caminos que profundizan la confrontación.

Como indicador cualitativo de diversos grados de conflictividad cabe mencionar, en primer lugar, la utilización de la violencia. Por ejemplo, la represión ejercida sobre trabajadores en huelga por la policía, el sistema judicial, grupos contratados por la patronal o una combinación de estos. Los asesinatos de Sacco y Vanzetti o de los ocho mártires de Chicago son los casos más conocidos pero no los únicos.

También los trabajadores en conflicto pueden utilizar la violencia para reprimir a los que no acatan la medida o contra la patronal; en Argentina y Uruguay durante la segunda mitad de la década de los años sesenta se registraron acciones armadas de apoyo a trabajadores en conflicto, como por ejemplo la toma de una empresa para leer una proclama o el secuestro de dueños de empresas en huelga.

Otro indicador cualitativo es la ocupación de la empresa, que los empresarios consideran un atentado al derecho de propiedad y los trabajadores la implementan como extensión del derecho de huelga. Un grado más es la puesta en marcha de las actividades por parte de los trabajadores. Por último, los indicadores cuantitativos utilizados por la OIT, en sus estadísticas de trabajo, son las horas de trabajo y el PBI perdidos por las interrupciones de actividades por medidas de lucha de las organizaciones sindicales.

\section{LAS RELACIONES DE PODER ENTRE LOS ACTORES}

Se percibe dentro de la empresa así como con relación al sistema político. Hyman (1989) analiza las relaciones de poder en la empresa, su relación con el papel del Estado y las alternativas estratégicas de las organizaciones sindicales. Señala que “... la desigualdad en el trabajo tiene una dimensión política, que está íntimamente conectada a su dimensión económica. ¿Las relaciones de trabajo deben ser autoritarias y antidemocráticas? ¿Son utópicas las aspiraciones de democracia industrial?" (HYMAN, 1989, p. 15 - traducción propia). Analiza la intervención del Estado y su incidencia en las relaciones de poder en el lugar de trabajo, así como el grado en que los trabajadores organizados pueden lograr mejores resultados por la presión o por la concertación con el Estado en la sociedad capitalista, así como el grado en que la debilidad económica de los trabajadores puede ser reducida por la legislación (Hyman, 1989).

Para lograr establecer las profundas implicancias del proceso que condujo a la degradación de las condiciones laborales es necesario comprender el espacio de trabajo como un campo de lucha, de dominación y resistencia, donde se conjugan, en un mismo movimiento, prácticas y representaciones en torno al trabajo (DROLAS, LENGUITA y MONTES, 2007, p. 7). 
Las estrategias empresariales de reorganización del trabajo "contribuyen a crear una representación simbólica que proyecta un trabajador aislado de toda construcción colectiva" (DROLAS, LENGUITA y MONTES, 2007, p. 7). Consideran que:

en ningún caso el profundo debilitamiento del mundo obrero se explica por motivos que se reducen únicamente a la dimensión económica, se justifica también por transformaciones importantes en las imágenes que se elaboran de ese mundo, en las formas en que se entablan las relaciones entre los trabajadores, con las empresas y con sus organismos de representación (DROLAS, LENGUITA y MONTES, 2007, p. 8).

Finalmente destacan la interrelación entre la dimensión económica y la política:

Así pues, las relaciones laborales constituyen un campo donde se ponen en juego dos órdenes de problemas: por un lado la lucha por los recursos, es decir la disputa en torno al salario - la determinación del plusvalor - . Por otra parte, en el campo laboral también se entabla la lucha por el establecimiento de las condiciones de trabajo en función de la organización de la producción, los ritmos y la productividad entre otros temas. Pero esta lucha que llevan adelante los diferentes sujetos que participan de las relaciones laborales también se dirime en el plano político, es decir en la capacidad que poseen los sujetos de imponer a los otros una visión, una lectura en torno a lo considerado adecuado, posible y deseado. Es por ello que resulta fundamental interpretar los acontecimientos sociales no como hechos, sino como producciones sociales, donde el poder actúa por medio de las condiciones objetivas y subjetivas de producción". La agenda en el plano microsocial definido por la empresa incluye la organización del trabajo, las políticas de recursos humanos, las calificaciones, las condiciones y medio ambiente de trabajo, las políticas de calidad total, el teletrabajo, como aspectos de las relaciones de poder. (DROLAS, LENGUITA y MONTES, 2007, p. 10)

Las condicionantes económicas inciden sobre las opciones estratégicas (traducción propia):

Aunque en este trabajo se subrayan los efectos de las estrategias empresariales, en diferentes condiciones ambientales tanto las estrategias de organizaciones de trabajadores como las del gobierno podrían servir como catalizadores del cambio. El argumento teórico desarrollado aquí puede ser válido para estos otros actores. (KOCHAN, MCKERSIE \& CAPPELLI, 1983, p. 19).

La probabilidad de las organizaciones sindicales de incidir en el proceso de toma de decisiones estratégicas de las empresas aumenta cuando la tasa de afiliación es alta y la negociación centralizada (Kochan, McKersie \& Cappelli, 1983), así como cuando mejora la situación económica, condicionantes que permiten negociar desde mejores posiciones de poder, tratar de lograr una mayor participación en las decisiones de la empresa o en el lugar de trabajo (Kochan, McKersie \& Cappelli, 1983).

\section{LAS PRINCIPALES CONDICIONANTES}

La pregunta central es: ¿Cómo se explica el perfil de los SRRLL en determinadas coordenadas de espacio y tiempo? Se puede desagregar en: ¿Cuáles son los criterios para 
identificar el origen, la permanencia y el cambio? La hipótesis es que las respuestas se pueden buscar en las interrelaciones con el contexto que modifica los componentes del sistema así como las relaciones entre ellos. Se consideran partes del contexto y condicionantes del SRRLL a la situación económica, la estructura social y el sistema político, incluyendo en este los aspectos jurídico-institucionales. Se incluyen las relaciones de poder en la empresa y con relación al sistema político.

el impacto del empleo y el cambio de su composición sobre las instituciones laborales no puede desdeñarse, ya que amplían su cobertura en sentido estricto. Pero la restitución de la vigencia de las instituciones laborales no se origina en ese impacto sino que fue sostenida en primer lugar políticamente y, en segundo lugar, socialmente, según un decurso temporal claramente perceptible (SENÉN, TRAJTENBERG y MEDWICK, 2010, p. 215 ).

Económicas - Se destacan tres aspectos económicos que impactan sobre el SRRLL en los países del Cono Sur de América Latina y definen líneas de investigación.

En primer lugar, la globalización y la apertura de las economías en un período de cambio tecnológico acelerado. Las ventajas comparativas que permitieron exportar a los países del Cono Sur durante la mayor parte del siglo veinte por la mano de obra relativamente barata para el nivel de calificación están doblemente jaqueadas: en los nuevos bienes y servicios, por las biotecnologías, la microelectrónica y la informática; en los bienes y servicios tradicionales, por el menor costo de mano de obra de la oferta competitiva de China e India. Es imprescindible invertir e incorporar tecnología, reducir costos, aumentar la calidad, diversificar la producción y los mercados.

En su exposición en el VI Congreso de ALAST realizado en abril de 2010, Novick (2010) destacó que el contexto internacional genera mayores incertidumbres y fluctuaciones, lo que exige a las empresas el permanente aumento de competitividad en el largo plazo y flexibilidad en la coyuntura.

Incide en aspectos jurídicos institucionales y “... la legislación laboral se ha ido abriendo a nuevas corrientes doctrinarias. El debate entre garantismo clásico, flexibilidad laboral y posiciones intermedias perdura, a veces con encono" (RELASUR, 1995, p. 26).

En segundo lugar, la integración regional de países con dimensiones económicas y situaciones muy distintas. En 2010, el PBI de Brasil se estimó en 1.823 miles de millones de dólares; Argentina, con 354.2 miles de millones, es la quinta parte de Brasil pero casi el doble que Chile y más de nueve veces Uruguay, con 41.8 (Área de Coyuntura IECON 2010, p. 31-43). Pero Brasil tiene el menor PBI por habitante, el mayor porcentaje de empleo informal urbano y el mayor porcentaje de población por debajo de la línea de pobreza. Con relación a los SRRLL se subraya que:

Los países de la región involucrados en el proceso de integración son tributarios de modelos de relaciones laborales y de ordenamientos jurídicos del trabajo que resultan singulares a cada Estado, pese a compartir algunos rasgos afines y comunes... Pese a esta diferenciación, corresponde señalar que el proceso integrador constituye un ámbito fértil para la configuración de un contexto regional con características propias, 
diferenciables de los escenarios nacionales, lo que comienza a visualizarse a través del desarrollo de propuestas y acciones comunes y conjuntas de los actores sociales. (ROSENBAUM, 2000, p. 9).

En tercer lugar, la situación y la política macroeconómica. En el transcurso de la década de los años noventa se percibió una progresiva debilidad de las organizaciones sindicales en todos los países del Cono Sur. Por una parte, es el reflejo de los cambios en la composición del producto y el empleo, con la pérdida de importancia relativa de la actividad industrial, el cierre o reducción de las grandes empresas y el aumento de los servicios prestados por empresas de pequeña escala (AOYAMA y CASTELLS, 2002). Por otra, es consecuencia de la retracción del Estado.

\section{Sociales}

\section{LOS ACTORES}

No existen actores sociales que puedan ser definidos por una sola dimensión y que sean representados por las fuerzas políticas. Todos los actores sociales son a la vez segmentados, multidimensionales y heterónomos. El análisis tiene entonces que desplazarse hacia el estudio del sistema político porque no existen clases u otras categorías integradas y fundamentales, que puedan ser estudiadas en sí, fuera de las intervenciones del poder político. (TOURAINE, 1987b, p. 84).

Los cambios en el mercado de trabajo y los actores generan nuevos problemas para los SRRLL. Para los ocupados en empleos atípicos, por informalidad o precariedad, o para los trabajadores por cuenta propia o del servicio doméstico, organizarse y tener representación es casi imposible. Para las empresas micro y pequeñas, negociar es difícil y caro y cuando existen negociaciones sectoriales, quedan representadas de hecho por las empresas grandes, sin que se manifiesten los matices de problemas e intereses, constituyendo un factor adicional de estímulo a la informalidad o al trabajo "en negro".

\section{LAS ESTRATEGIAS}

La reestructura de la producción y el comercio, con actividades que se expanden y otras que se contraen, modifica las oportunidades de empleo: las nuevas no coinciden con las viejas, por empresa, rama, calificación o región. En la empresa y a nivel de sectores será necesario enfrentar el desempleo que se incrementa en determinadas actividades, regiones o calificaciones, negociar las reducciones de personal, orientar su reinserción y las compensaciones. Se vuelve imprescindible una negociación a nivel de cada empresa para operar a favor del aumento de la competitividad y la generación de empleo. Los programas de capacitación para adquirir las nuevas calificaciones que demanda el cambio tecnológico adquieren un carácter prioritario.

El contexto económico enfrenta a los actores a nuevas opciones y se requiere que los actores sociales tengan vocación de negociación, así como una jerarquización de lo que quieren lograr y lo que pueden ceder. Pueden continuar con las prácticas anteriores centrando las 
negociaciones y los conflictos en el salario, buscando comprimirlo por parte de la empresa para responder a las nuevas exigencias de competitividad o tratando de aumentarlo por parte de los trabajadores para evitar el deterioro o mejorar su poder de compra ${ }^{(4)}$. Una segunda posibilidad es la búsqueda de una respuesta nueva que exige a ambas partes una perspectiva diferente.

\section{Políticas}

Se analiza cómo condiciona el sistema político a los actores sociales, tanto en su estructura y desarrollo como en sus estrategias. En parte se ha visto con el papel del Estado en el Sistema. No se puede afirmar, a priori, que la militancia o la definición partidaria de los dirigentes empresariales o sindicales impida o limite su independencia como dirigentes de una organización que expresa determinado punto de vista e intereses. Se requiere investigación empírica.

Por otra parte, no se podría negar que las definiciones partidarias de los dirigentes son el resultado de un sistema de valores que condicionan sus puntos de vista en su actividad en las organizaciones, desde las reivindicaciones y las medidas de lucha, hasta los criterios para valorar éxitos y fracasos. Ejemplos muy claros fueron las medidas de lucha empresariales contra el gobierno de Salvador Allende en Chile, el paro de las organizaciones agropecuarias contra el gobierno de Cristina F. de Kirchner en la Argentina o la huelga general de la central sindical uruguaya contra el golpe de estado en 1973.

La inclusión social y política de la clase trabajadora en América Latina se ha instrumentado principalmente por la regulación del mercado de trabajo como parte del desarrollo industrial de sustitución de importaciones (Cardoso y Gindin, 2009). Se señala a Argentina, Brasil, México, Perú y Venezuela como casos típicos de simbiosis entre desarrollo y control del Estado sobre las fuerzas sociales emergentes impulsadas por el proceso.

A fines de la década de los ochenta, Touraine (1987b) diferencia tres situaciones en la relación Estado-sindicatos:

En primer lugar, el sindicalismo sin organización independiente como es el caso brasileño, y en gran parte el mexicano. En segundo lugar, los países de central sindical única que son, en particular, Uruguay, Chile, Venezuela y Bolivia. En tercer lugar los países con pluralismo sindical paralelo al pluralismo político, lo que es el caso de Colombia, Perú y en parte, Ecuador. ¿Cómo se explican tales diferencias? No por las ideas de los sindicalistas o por la influencias de ciertos partidos políticos, sino más bien por la naturaleza del sistema político mismo. En México y Brasil existe un Estado fuertemente intervencionista - incluso antes del período de dictadura militar en Brasil - que incorpora a los sindicatos como a los empresarios en su proyecto de transformación de la sociedad. Tal fue también el caso de Argentina en el período de Perón (1945-1955) (TOURAINE, 1987b, p. 206).

(4) Algunos empresarios agregan la persecución y algunos sindicatos la confrontación. Cabe preguntarse cuál es el origen del proceso y cómo se retroalimentan las decisiones, qué importancia relativa tienen y cómo condicionan la viabilidad de la mayor cooperación. 
Con el retorno a la democracia de los países del Cono Sur, el Programa Regional del Empleo para América Latina y el Caribe (PREALC) de OIT analizó la viabilidad y el papel de la concertación de políticas de empleo e ingresos entre los actores sociales y los gobiernos, considerando que era un instrumento de consolidación de la democracia (PREALC, 1988). A principios del siglo XXI Cardoso y Gindin (2009, p. 40) concluyen que el nuevo contexto económico y político ayudó a fortalecer la posición de los sindicatos en las negociaciones en Argentina y Brasil.

\section{LOS PRINCIPALES IMPACTOS}

Los componentes del perfil del SRRLL impactan sobre el contexto económico, social y político; las características de los actores, de los escenarios, del grado de cooperación y confrontación así como de las relaciones de poder se proyectan más allá del sistema. La interrogante central es: ¿contribuye a una estrategia de crecimiento con aumento de competitividad, de empleo de calidad y de salarios?

Los resultados económicos para los trabajadores se miden en términos de empleo y salarios; para las empresas, en términos de competitividad, rentabilidad y clima de inversión. En el plano social, de acuerdo al nacimiento, desarrollo, fortalecimiento o desaparición de los actores. Los resultados políticos, por los cambios en la distribución de poder entre los actores en la empresa y por la relación con el sistema político. Un problema clásico es la relación entre los objetivos económicos y los políticos, las estrategias que los consideran complementarios y las que los consideran contradictorios, y la opción se refleja en los diversos grados de combinación de cooperación y confrontación.

\section{ECONÓMICOS}

En este contexto, las instituciones de las Relaciones Laborales, como mecanismo de inclusión, ayudaron a pavimentar el camino para el proceso de desarrollo económico basado en el modelo ISI y promovido por Estados variados, autoritarios, populistas y democráticos (CARDOSO y GINDIN, 2009, p. 1 - traducción propia).

Se subrayó que:

Si los dos factores clave del desarrollo - empleadores y trabajadores - carecen de motivación necesaria, es indudable que no se alcanzarán las tasas de capitalización e inversiones necesarias, ni tampoco la expansión del mercado interno, la cooperación y las mejoras de la productividad que caracterizan un desarrollo acelerado. Por otra parte, no es posible concebir una coordinación adecuada entre las múltiples operaciones que se realizan en una empresa sin que haya entendimiento entre la administración y el personal. Tampoco es factible prever un desarrollo efectivo a nivel macroeconómico, sin que exista una voluntad común de llevarlo adelante, un cierto consenso sobre las estrategias que se han de seguir y un entendimiento claro sobre la justa distribución de sus beneficios (OIT, 1987, p. 1). 
Se establece que el SRRLL debe cumplir cuatro cometidos: fijar las condiciones sustantivas de trabajo, regular las relaciones entre los interlocutores, resolver el conflicto social y promover la participación y la cooperación.

El desarrollo de las relaciones laborales en América Latina se fundamenta como condición de la generación de empleo y se explica por la transformación productiva que:

implica el surgimiento de una nueva empresa, donde se generan mayores necesidades y oportunidades de colaboración entre empresarios y trabajadores, se abren nuevos espacios de diálogo y negociación y se redefinen algunos aspectos de las políticas laborales (OIT, 2003, p. 35).

Los impactos de las estrategias sindicales en América Latina fueron analizados por varios autores y editados por Kuhn y Márquez (2005). En el sector privado analizaron la relación entre el grado de competencia y la tasa de afiliación sindical en la industria manufacturera de Brasil, Perú y Uruguay, tratando de probar la hipótesis de que uno de los principales motivos de la afiliación es lograr una transferencia de rentas desde los propietarios de las empresas y los consumidores; el supuesto implícito es que sin los sindicatos no habría transferencias de rentas en el sentido contrario y se desconocen las relaciones entre tipo de cambio, precios y salarios en ese proceso. En el sector público se analizaron las organizaciones de los maestros en Argentina y Perú.

Las preguntas orientadoras son: ¿qué resultados impuso el contexto y cuál fue el papel de las opciones estratégicas de los actores así como de sus interrelaciones? Se trata de identificar el margen de libertad que dispusieron y cómo lo utilizaron, cómo eligieron y por qué.

\section{Sociales}

Los SRRLL que logran canalizar los conflictos promoviendo una cultura de cooperación se han convertido en un poderoso instrumento para la reestructura de las empresas que buscan una mayor competitividad (Ozaki, 2003).

Una estrategia dirigida al aumento de la competitividad y la productividad

"debe considerar la formulación de normas laborales y contratos colectivos que tengan como objetivo propiciar la mayor estabilidad a los trabajadores, inversiones en las personas y sistemas de remuneración que promuevan la productividad y la calidad, como por ejemplo: adicionales por desempeño, por méritos, además de obedecer a criterios de justicia salarial" (CACCIAMALI, 2002, p. 18).

Las empresas que perciben la necesidad de aumentar la competitividad tienen que poner en marcha cambios tecnológicos y en la organización del trabajo. Como problema de relaciones laborales la pregunta es: ¿ ¿los cambios se procesan con el sindicato, sin el sindicato o contra el sindicato? La experiencia muestra ejemplos de las tres situaciones.

Para la empresa, la estrategia de negocios implica un enfoque de las relaciones laborales, distintas políticas de personal y respuestas a las reivindicaciones. Por ejemplo, una estrategia exportadora con inversión y cambio técnico requiere trabajadores de mayor calificación, 
polifuncionales, actividad continua etc. Como contrapartida será necesario mejorar el acceso a la información, crear organismos de cooperación, estimular la productividad, acceder a fórmulas de seguridad en el empleo. Se reflejará en la mayor importancia a la administración de Recursos Humanos y Relaciones Laborales, en general, por la creación de una gerencia. La clave es la forma en que se toman las decisiones que afectan a los trabajadores, así como los procedimientos de consulta y negociación.

Se abren nuevas opciones para las estrategias sindicales: aceptar los cambios o rechazarlos en bloque aumentando la conflictividad; manejar la opción empleo-ingresos; aceptar costos presentes negociando beneficios futuros. Los cambios en la organización y las condiciones de trabajo que reclaman las nuevas tecnologías pueden tener como consecuencia aumentar las dificultades de sindicalización, poniendo frente a un dilema a la acción sindical (Martín Artiles, 1994). Esta a su vez se enmarca en objetivos de corto plazo, económicos, o de mediano y largo plazo, políticos. En esta segunda opción, los objetivos políticos pueden inscribirse en una estrategia electoral o insurreccional.

Los puntos de vista de la administración de la empresa y de los trabajadores son contradictorios, en la medida que tienen objetivos económicos prioritarios distintos, pero no antagónicos, ya que se pueden lograr simultáneamente. La búsqueda de una mayor competitividad por la reducción de los salarios reales o la búsqueda de mayores niveles de salarios por el aumento de precios, son soluciones conflictivas. Del mismo modo son conflictivos el ajuste por la reducción del número de ocupados o la aspiración sindical de mantener sin cambios los puestos, las tareas y las calificaciones. Las remuneraciones variables pueden resolver estos conflictos, motivan un mejor desempeño, comparten los resultados de la empresa y flexibilizan el costo de mano de obra. El problema es más complejo en la consideración de los objetivos políticos.

Se puede investigar tres formas de relación de los actores sociales con el Estado en las políticas públicas:

a) Demandar acciones al sistema político que les reporten beneficios y descarguen los costos sobre otros sectores de la sociedad. Se trata de incidir en el ejercicio del poder, modificar la acción del gobierno o generar costos políticos. Predominantemente las reivindicaciones sindicales contribuyen a la redistribución del ingreso y las empresariales a la concentración.

b) Cuando existen los escenarios adecuados para el Diálogo Social, los actores pueden contribuir en el diseño identificando problemas y puntos de vista sobre las posibles soluciones, así como en la ejecución y el control de las acciones.

c) Diseñar e implementar acciones con autonomía, que predominantemente se acuerdan en convenios colectivos. Requiere un marco jurídico que lo valide.

\section{REFERÊNCIAS BIBLIOGRÁFICAS}

AGHION, Philippe; ALGAN, Yann; CAHUC, Pierre. Can policy interact with Culture? Minimum wages and the Quality of Labor Relations. Washington: [World Bank] 2008. 
AOYAMA, Yuko; CASTELLS, Manuel. Estudio empírico de la sociedad de la información. Composición del empleo en los países del G7 de 1920 a 2000. In: Revista Internacional del Trabajo, v. 121, n. 1-2, 2002.

ARRIGO, Gianni; CASALE, Giuseppe (editores generales). Glossary of labour, law and industrial relations (with special reference to the European Union). Ginebra: ILO, 2005.

BLANCHARD, Olivier; PHILIPPON, Thomas. The quality of labor relations and unemployment, MIT Department of Economics Working Paper 04-25,2004. Disponible en: <http://ssrn.com/ abstract $=559203>$ or $<$ doi:10.2139/ssrn.559203> Acceso en: $1^{\circ}$ jul. 2010.

BRAVERMAN, Harry. Trabajo y capital monopolista. La degradación del trabajo en el siglo XX, Nuestro Tiempo, México, 1987.

CACCIAMALI, Maria Cristina. Liberalización del comercio y desafíos para implementar los principios y derechos fundamentales del trabajo en América Latina. In: CACCIAMALI, Banko, C.; KON, A. Los desafios de la política social en América Latina. Caracas, 2002.

CARDOSO, Adalberto; GINDIN, Julián. Industrial relations and collective bargaining: Argentina, Brazil and Mexico compared. Ginebra: ILO, 2009.

DE LA GARZA, Enrique. Fin del trabajo o trabajo sin fin. In: DE LA GARZA, E. (Coord.). Tratado latinoamericano de sociología del trabajo. Ciudad de México: Fondo de Cultura Económica, 2000, p. 755-774.

. Exposición en la mesa redonda "Antiguos y nuevos trabajos: ¿Hacia un concepto ampliado de trabajo?”. VI Congreso de ALAST, abr., México, 2010.

DROLAS, Ana; LENGUITA, Paula; MONTES CATÓ, Juan (Comps.). Relaciones de poder y trabajo: las formas contemporáneas de explotación, Buenos Aires: Poder y Trabajo Editores, 2007.

DUNLOP, John Thomas. Industrial relations systems. Nueva York: Henry Holt, 1958.

GARCÍA, Norber to E. Productividad, competitividad y empleo: un enfoque estratégico, 2002. Disponible en: <www.consorcio.org/CIES> Acceso en: 1ํ jul. 2010.

GODIO, Julio; VILLARROEL, Amalia. Curso de relaciones laborales. Buenos Aires: Universidad de Lomas de Zamora, 2007.

Post Scriptum a el industrialismo y el hombre industrial. In: Revista Internacional del Trabajo, v. 83, n. 6, Ginebra, 1971.

KOCHAN, Thomas; KATZ, Harry. Collective bargaining and industrial relations. Nueva York: Irwin, Homewood Ill, 1988.

KOCHAN, Thomas; KATZ, Harry; MAC KERSIE, Robert. La transformación de las relaciones laborales en los Estados Unidos, MTSS. España, 1993.

KOCHAN, Thomas; LOCKE, Richard; PIORE, Michael. (Eds.). Employment relations in a changing world economy. Cambridge: MIT Press, 1995.

KOCHAN, Thomas; MC KERSIE, Robert; CAPPELLI, Peter. Strategic choice and Industrial Relations Theory and Practice, SSP WP n. 1506-83, 1983.

KOCHAN, Thomas; PIORE, Michael. Proposal for comparative research on industrial relations and human resource policy and practice. Reunión de expertos, OCDE, París, 1990.

KUHN, Peter; MÁRQUEZ, Gustavo. What difference do Inions make?, Washington: BID, 2005.

MARTÍN ARTILES, A. Flexibilidad y relaciones laborales. Estrategias empresariales y acción sindical. Universidad Autónoma de Barcelona, 1994. 
NOVICK, Marta. Globalización, el mundo del trabajo y el empleo. La experiencia latinoamericana. Exposición en la mesa redonda. La globalización y sus consecuencias sobre el trabajo en América Latina. VI Congreso de ALAST, 2010.

OIT. Algunos aspectos de las relaciones de trabajo en América. Ginebra, 1962.

Las relaciones colectivas de trabajo en América Latina. Ginebra, 1981.

Relaciones de trabajo en el Uruguay. Serie Relaciones de Trabajo 66, Ginebra, 1987.

. Políticas de empleo en Chile y América Latina. Seminario en honor de Víctor E. Tokman, OIT, Santiago, 2003.

MARTÍNEZ MARTíN, Rafael; PRIOR RUIZ, Juan Carlos (Coords.) El trabajo en el siglo XXI. Granada: Editorial Comares, 2005.

OZAKI, Muneto. Negotiating job protection in the age of globalization. Ginebra: ILO, 2003.

RELASUR. Las relaciones laborales en el Cono Sur. Estudio comparado. Madrid: OIT-MTSS, 1995.

ROSENBAUM RIMOLO, Jorge. Negociación colectiva sobre formación en el MERCOSUR. Montevideo: OIT/CINTERFOR, 2000.

SENÉN, Cecilia; TRAJTEMBERG, David; MEDWID, Bárbara. La determinación del nivel de negociación colectiva en la Argentina. ¿Una vuelta hacia la centralización? Ponencia presentada en el VI Congreso de ALAST, México, abr. 2010.

Actores sociales y sistemas políticos en América Latina. Santiago: PREALC, 1987b. 